Short communication

\title{
Synchrotron radiation microbeam $X$-ray fluorescence analysis of zinc concentration in remineralized enamel in situ
}

\author{
Tsunenori Matsunaga ${ }^{a}$, Hidetaka Ishizaki ${ }^{a}$, Shuji Tanabe ${ }^{b}$, Yoshihiko \\ Hayashi $^{a, *}$
}

${ }^{a}$ Department of Cariology, Nagasaki University Graduate School of Biomedical Sciences, Sakamoto 1-7-1, Nagasaki 852-8588, Japan

${ }^{b}$ Department of Materials Science, Nagasaki University Graduate School of Science and Technology, Bunkyo-machi 1-14, Nagasaki 852-8521, Japan

*Corresponding author. Tel: +8195 819 7677; fax: +81958197680.

E-mail address: hayashi@nagasaki-u.ac.jp(Y. Hayashi) 


\section{ABSTRACT}

Objective: Remineralization is an indispensable phenomenon during the natural healing process of enamel decay. The incorporation of zinc ( $\mathrm{Zn}$ ) into enamel crystal could accelerate this remineralization. The present study was designed to investigate the concentration and distribution of $\mathrm{Zn}$ in remineralized enamel after gum chewing.

Methods: The experiment was performed at the Photon Factory. Synchrotron radiation was monochromatized and $\mathrm{X}$-rays were focused into a small beam spot. The X-ray fluorescence (XRF) from the sample was detected with a silicon Si(lithium (Li)) detector. X-ray beam energy was tuned to detect Zn. The examined samples were small enamel fragments remineralized after chewing calcium phosphate-containing gum in situ. The incorporation of Zn atom into hydroxyapatite (OHAP), the main component of enamel, was measured using $\mathrm{Zn}$ K-edge extended X-ray absorption fine structure (EXAFS) with fluorescence mode at the SPring-8.

Results: A high concentration of $\mathrm{Zn}$ was detected in a superficial area $10 \mu \mathrm{m}$ deep of the sectioned enamel after gum chewing. This concentration increased over that in the intact enamel. The atomic distance between $\mathrm{Zn}$ and $\mathrm{O}$ in the enamel was calculated using the EXAFS data. The analyzed atomic distances between $\mathrm{Zn}$ and $\mathrm{O}$ in two sections were 0.237 and $0.240 \mathrm{~nm}$ Conclusion: The present experiments suggest that $\mathrm{Zn}$ is effectively incorporated into remineralized enamel through the physiological processes of mineral deposition in the oral cavity through gum-chewing and that $\mathrm{Zn}$ substitution probably occurred at the calcium position in enamel 
hydroxyapatite.

Keywords: Enamel, Remineralization, Zinc, Synchrotron radiation, X-ray fluorescence

\section{Introduction}

Many trace elements were incorporated in the biologically mineralized tissues. ${ }^{1,2}$ In particular, $\mathrm{Zn}$ is a dietary essential trace element known to be necessary for normal collagen synthesis and mineralization of bone. ${ }^{3,4}$ It has long been said that zinc compounds, such as zinc chloride and zinc citrate are beneficial for teeth, and that zinc oxide inhibits dentine demineralization. ${ }^{5}$ However, the mechanisms by which Zn inhibits dentine demineralization are not fully resolved. In men, dietary Zn intake and plasma Zn both have a positive relationship to bone mineral density. ${ }^{6} \mathrm{Zn}$ has also been demonstrated to have a potent stimulatory effect on osteoblastic bone formation ${ }^{7,8}$ and an inhibitory effect on osteoclastic bone resorption. ${ }^{9,10}$ Furthermore, Zn can stimulate protein synthesis in osteoblastic cells in vitro by active aminoacyl-tRNA synthetase. ${ }^{11} \mathrm{Zn}$ acts as a nucleator in the formation of the whitlockite phase in vitro. ${ }^{12}$ The $\mathrm{Zn}$ incorporation in HA and whitlockite have also been investigated in in vitro conditions. ${ }^{13,14}$ The $\mathrm{Zn}$ content decreased significantly from the outer to the inner enamel. ${ }^{15}$ However, the exact distribution and concentration of $\mathrm{Zn}$ are still obscure in enamel remineralization. 
The incorporation of $\mathrm{Zn}$ into enamel crystal could promote this remineralization. The present study was designed to investigate the $\mathrm{Zn}$ incorporation and concentration in a remineralized enamel surface. The experiment was performed by synchrotron radiation induced X-rays focused into a small beam spot. The XRF from the sample was detected with a Si(Li) detector. For the purpose of trace element detection, synchrotron radiation analyses bring sub-micron resolution and detection limits of the ppm range. We hypothesized that the distribution and concentration of $\mathrm{Zn}$ in mineral deposition could be elucidated using calcium-containing chewing gum to accelerate the remineralization. Thereafter, the local environment of $\mathrm{Zn}$ atom in remineralized enamel was analyzed by using the XAFS techniques.

\section{Materials and methods}

\subsection{Sample preparation}

Three third molars with a clinical diagnosis of pericoronitis without carious lesions were extracted with the patient's consent. After extraction, the enamel of the coronal region, about $1 \times 1 \times 1 \mathrm{~mm}$ was excised with a diamond disk under a stream of Ringer's solution. After coating 5 planes of these cubic fragments except the original enamel surface with nail varnish, artificial demineralization buffer which contained $0.1 \mathrm{M}$ lactic acid, $3.0 \mathrm{mM}$ $\mathrm{CaCl}_{2}, 1.8 \mathrm{mM} \mathrm{KH}_{2} \mathrm{PO}_{4}$ was used to create artificial enamel lesions for 3 days at $37^{\circ} \mathrm{C}, \mathrm{pH}$ 4.5. After demineralization, these fragments were fully rinsed with tap water and distilled water and dried at room temperature for 1 week. Afterward, these fragments were sterilized using low temperature 
plasma sterilization

\subsection{Oral appliance preparation}

Three demineralized enamel fragments were set on the occlusal plane of each appliance which was made of acrylic resin. These appliance were set in the 11 volunteers' (no smokers, at least 28 natural teeth with no current caries activity and periodontal disease, no antibiotic medication, with $1 \mathrm{~mL} / \mathrm{min}$ of salivary flow rate) oral cavity for remineralization experiments through gum

chewing in situ. ${ }^{16}$ Approval for this study was obtained from the Nagasaki University Graduate School of Biomedical Sciences Human Research Committee, and all subjects provided their writtem informed concent.

\subsection{Remineralization experiment in situ}

Two different types of calcium phosphate-containing xylitol chewing gums on the market were examined with at least one week as a rest period between treatments. One was Xylitol ${ }^{\circledR}$ (Lotte Co.; L gum), which contained xylitol, funoran and calcium hydrogen phosphate. The other was Poscam ${ }^{\circledR}$ (Glico Co.; G gum), which contained xylitol and calcium phosphorylated oligosaccharide. For the pellet gum study, the subjects set the appliance in the mouth chewed for 10 minutes and the appliances were retained for another 10 minutes in a subject's mouth; 5 times daily for seven days (sufficient for quantitative comparison using energy dispersive X-ray microanalysis $(\mathrm{EDX})) .{ }^{16}$ This in situ study was carried out by the double blind method.

\subsection{Specimen preparation}

After the completion of each treatment, the enamel pieces were removed 
from the appliances, fixed and dehydrated in ethanol. The ethanol was replaced with $t$-butanol and the specimens were freeze-dried and preserved in the container until analysis. For SEM and EDX of the enamel sections, each enamel fragment was divided into two pieces using a razor blade. One piece of enamel section with relatively smooth surface was used for the $\mathrm{Zn}$ analysis without carbon coating.

\subsection{Synchrotron experiment}

The experiment was performed on a beam line (BL)-4A (the station for XRF analyses) at the Photon Factory, High Energy Acceleration Research organization (KEK) in Tsukuba, Japan. Synchrotron radiation from a bending magnet was monochromatized using a synthetic multiplayer monochromator and X-rays were focused into a small beam spot of about $4 \mu \mathrm{m}$ diameter using a Pt-coated ellipsoidal mirror. The sample was moved by an $x-y-z$ stepping motor $(1 \mathrm{~mm})$ while being observed by the optical microscope. The XRF from the sample was detected with a $\mathrm{Si}(\mathrm{Li})$ detector (Princeton Gamma-Tech. Inc., Princeton, NJ, USA). X-ray beam energy was tuned to be $14.2 \mathrm{keV}$ to detect $\mathrm{Zn}$. Zn spectrum was analyzed at 6 points, the $10 \mu \mathrm{m}$ step of interval from the superficial layer ( $0 \mu \mathrm{m}$ deep) to the inner layer (50 $\mu \mathrm{m}$ deep) and in 5 files (100 $\mu \mathrm{m}$ interval) in each piece. A total of 59 pieces $\{18$ pieces for L gum, 23 pieces for G gum, 4 pieces for control without gumchewing (C), 7 pieces for only demineralization (D) and 7 pieces for intact enamel (I)\} were measured. The standard reference material (SRM) was used during the calibration process. The area density of each spot was precisely determined by comparing it to that of a reference thin film. SRM consists of 
a $64 \mu \mathrm{g} / \mathrm{cm}^{2}$ density of zinc which has deposited on the Mylar membrane (2.5 $\mu \mathrm{m}$ thick) (Micromatter Co., Deer Harbor, WA, USA).

Furthermore, the possibility of substitution of $\mathrm{Zn}$ atom into OHAP as a main component of enamel crystal was preliminarily analyzed in 2 specimens (one remineralized and one intact pieces) using $\mathrm{Zn}$ K-edge EXAFS with fluorescence mode on BL37XU (the station for XRF analyses) at the largest synchrotron radiation facility in the world (SPring-8, Hyougo, Japan). X-ray microbeam ( $1 \mu \mathrm{m}$ diameter) energy was tuned to be $10 \mathrm{keV}$ to detect $\mathrm{Zn}$. Three points were measured in each piece at 4-5 $\mu \mathrm{m}$ deep from surface. A 5\% Zn-substituted OHAP powder was used for the reference. ${ }^{17}$ The EXAFS signals were analyzed using a special software program (REX2000, Rigaku Co., Japan).

\subsection{Statistical analysis}

Each Zn concentration was expressed as the mean \pm SE. Paired Student's t-test was applied to assay any significant difference among the data measured. Significance was set at a probability level of $p<0.05$.

\section{Results}

The concentration of $\mathrm{Zn}$ was highest at the superficial layer in all samples (Table 1). Then, the statistical comparison of $\mathrm{Zn}$ distribution was carried out using the average concentration of both 0 and $10 \mu \mathrm{m}$ deep areas from surface. The remineralized enamel outer layer after $\mathrm{L}$ gum chewing revealed the highest Zn concentration, which showed also significantly higher than that in intact enamel $(p<0.05)$. There was no significant difference between $G$ 
gum chewing and the intact enamel groups. The $\mathrm{Zn}$ concentration in the remineralized enamel after either $\mathrm{L}(p<0.01)$ or $\mathrm{G}(p<0.05)$ gum chewing was significantly higher than that in the control group without gum chewing. The average $\mathrm{Zn}$ concentration in the remineralized enamel after chewing the two types of gum was not significantly higher than that in the intact enamel.

The atomic distance between $\mathrm{Ca}$ and the nearest $\mathrm{O}$ in OHAP is theoretically $0.258 \mathrm{~nm} .{ }^{18,19}$ The difference of an ionic radius between Ca and $\mathrm{Zn}$ is about $0.02 \mathrm{~nm}$. Then, $0.238 \mathrm{~nm}(0.258$ minus 0.02$)$ was set up as the atomic distance between $\mathrm{Zn}$ and $\mathrm{O}$ in OHAP for the software analyses. The EXAFS data analyses showed that the average atomic distance between $\mathrm{Zn}$ and $\mathrm{O}$ was calculated to be $0.237 \pm 0.004 \mathrm{~nm}$ in one piece of remineralized enamel and $0.240 \pm 0.000 \mathrm{~nm}$ in one piece of intact enamel.

\section{Discussion}

This study is the first report that $\mathrm{Zn}$ is present in the superficial layer of remineralized enamel after gum chewing, and is consistent an earlier study in the intact enamel. ${ }^{15}$ The highest concentration of $\mathrm{Zn}$ was detected in the superficial layer of the enamel section. We have already confirmed that EDX showed a tendency for high $\mathrm{Ca} / \mathrm{P}$ molar ratio in the superficial layer of remineralized enamel. ${ }^{16}$ The present study also shows that gum chewing promotes the $\mathrm{Zn}$ accumulation into the surface of remineralized enamel. These findings strongly suggest that $\mathrm{Zn}$ is closely related to the mineralization process. 
The present data indicate that calcium phosphate-containing gum chewing for 1 week could restore the $\mathrm{Zn}$ concentration in the artificial demineralized enamel. This means that the incorporation of $\mathrm{Zn}$ originated from saliva into demineralized enamel is probably necessary for the remineralization phenomena and that the gum chewing could promote the remineralization process in enamel. $\mathrm{Zn}$ is thought to be an important and indispensable trace element for mineral deposition in enamel remineralization, as it is in osteoblastic bone formation. ${ }^{7,8}$ Although further studies must be conducted to determine the interaction between $\mathrm{Zn}$ and $\mathrm{Ca}$ ions, this study supports the possibility that $\mathrm{Zn}$ is an indispensable trace element for tooth mineralization, especially the acceleration of mineralization.

This study is also the first XAFS examination of remineralized enamel. The analyzed atomic distances between $\mathrm{Zn}$ and $\mathrm{O}$ in two sections were 0.237 and $0.240 \mathrm{~nm}$, which were very close to the theoretically shortest distance of $0.238 \mathrm{~nm}$. However, the atomic distance between $\mathrm{Zn}$ and $\mathrm{O}$ in the standard zinc oxide crystal is $0.195-0.198 \mathrm{~nm}$, whose distance is completely different from the present distance. The negative correlations show the possibility of substitutions among the elements Ca-Zn for the different types of teeth and dental mineral. ${ }^{13,20}$ Although the present experiments for XANES and EXAFS was done using very limited specimens, the present data suggest that $\mathrm{Zn}$ should be incorporated into remineralized enamel through the gum chewing process for mineral deposition and that $\mathrm{Zn}$ substitution probably occurred at the calcium position in the hydroxyapatite crystal of remineralized enamel as in the superficial layer of an intact enamel . 


\section{REFERENCES}

1. Ainsworth T. Application of PIXE to studies in dental and mental healths. Nuc Instrum Meth 1981;181:333-6.

2. Chaudhri MA. Nuclear analytical methods in calcified tissue research. Nutrition 1995;11:538-41.

3. Calhoun NR, Smith Jr JC, Becker KL. The role of zinc in bone metabolism. Clin Orthop 1974;103:212-34.

4. Suwarnasarn A, Wallwok JC, Lykken GI, Low FN, Sandstead HH. Epiphyseal plate development in the zinc-deficient rat. $J$ Nutr 1982;112:1320-8.

5. Takatsuka T, Hirano J, Matsumoto H, Honma T. X-ray absorption fine structure analysis of the local environment of zinc in dentine treated with zinc compounds. Eur J Oral Sci 2005;113:180-183.

6. Hyun TH, Barrett-Connor E, Milne DB. Zinc intakes and plasma concentrations in men with osteoporosis: the Rancho Bernardo Study. Am J Clin Nutr 2004;80:715-21.

7. Yamaguchi M, Oishi H, Suketa Y. Stimulatory effect of zinc on bone formation in tissue culture. Biochem Pharmacol 1987;36:4007-12.

8. Hashizume M, Yamaguchi M. Stimulatory effect of $\beta$-alanyl-L-histidinato zinc on cell proliferation is dependent on protein synthesis in osteoblastic MC3T3-E1 cells. Mol Cell Biochem 1994;122:59-64.

9. Kishi S, Yamaguchi M. Inhibitory effect of zinc compounds on osteoclast-like cell formation in mouse marrow cultures. Biochem 
Pharmacol 1994;48:1225-30.

10. Yamaguchi $M$, Uchiyama S. Receptor activator of NF- $\kappa B$ ligand-stimulated osteoclastogenesis in mouse marrow culture is suppressed by zinc in vitro. Int J Mol Med 2004;14:81-5.

11. Yamaguchi M, Kishi S, Hashizume M. Effect of zinc-chelating dipeptides on osteoblastic MC3T3-E1 cells: Activator of aminoacyl-tRNA synthase. Peptides 1994;15:1367-71.

12. Knuuttila M, Lappalainen R, Kontturi-Narhi V. Effect of Zn and MG on the formation of whitlockite in human subgingival calculus. Scand J Dent Res 1980;88:513-6.

13. Ingram GS, Horay CP, Stead WJ. Interaction of zinc with dental mineral. Caries Res 1992;26:248-53.

14. Davey HP, Embery G, Cummins D. Interaction of zinc with a synthetic calcium phosphate mineral. Caries Res 1997;31:434-40.

15. Frank RM, Sargentini-Maier ML, Turlot JC, Lerov MJ. Zinc and strontium analyses by energy dispersive X-ray fluorescence in human teeth. Arch Oral Biol 1989;34:593-7.

16. Hayashi Y, Matsunaga T, Viloria LI. X-ray microanalysis of remineralized enamel lesions by xylitol-containing chewing gums having different types of calcium phosphate. Jpn J Conserv Dent 2005;48:648-55. [in Japanese].

17. Tieliewuhan Y, Hirata I, Okazaki M, Takatsuka T, Matsumoto H, Honma T. Crystallographic properties of Zn-containing hydroxyapatites. In: Okazaki M et al. ed. Archives of Bioceramic Research, Vol. 3. Proceedings 
of the 3rd Asian Bioceramics Symposium in Conjunction with Hard Tissues Nano-Biomaterials Symposium. Saga, Japan: Saga Printing Ltd, 2003; 263-6.

18. Kay MI, Young RA. Crystal structure of hydroxyapatite. Nature 1964;204:1050-2.

19. Okazaki M, Sato M. Computer graphics of hydroxyapatite and $\beta$-tricalcium phosphate. Biomaterials 1990;11:573-8.

20. Gurguera E, Romero Z, Burguera M, Burguera JL, de Arenas H, Rondon $C$, et al. Determination of some cationic species in temporary teeth. $J$ Trace Elem Med Biol 2002;16:103-12. 
Table 1 - Zn concentration (ppm) at the superficial layer in each group

\begin{tabular}{|c|c|c|c|c|c|}
\hline$\frac{\text { Group }}{\mu_{\text {deep }}}$ & $\mathrm{L}$ & G & $\mathrm{C}$ & $\mathrm{D}$ & I \\
\hline 0 & $777.70 \pm 32.44$ & $696.96 \pm 15.17$ & $611.60+28.15$ & $506.97 \pm 20.55$ & $643.32 \pm 20.57$ \\
\hline 10 & $673.15 \pm 24.71$ & $577.04 \pm 17.22$ & $481.54 \pm 24.19$ & $434.98 \pm 16.68$ & $550.69 \pm 24.10$ \\
\hline A & $725.42+21.95$ & $637 \pm 14.44$ & $543.57 \pm 29.99$ & $470.98 \pm 16.17$ & $597.00 \pm 19.92$ \\
\hline 20 & $577.83 \pm 25.85$ & $484.64 \pm 18.44$ & $396.64 \pm 30.65$ & $377.89 \pm 21.77$ & $484.78+24.98$ \\
\hline 30 & $499.68 \pm 29.50$ & $419.48+18.69$ & $344.13 \pm 38.60$ & $330.06 \pm 25.62$ & $436.83+23.18$ \\
\hline 40 & $434.19 \pm 30.84$ & $370.67 \pm 18.96$ & $317.78+40.15$ & $290.22 \pm 26.60$ & $399.83 \pm 19.86$ \\
\hline 50 & $380.07 \pm 29.00$ & $330.36 \pm 17.56$ & $295.35+40.84$ & $258.91 \pm 25.85$ & $365.43 \pm 15.83$ \\
\hline
\end{tabular}

A, the average concentration of both 0 and $10 \mu \mathrm{m}$ deep areas; C, control without chewing; D, only demineralized enamel; I, intact enamel. 